\title{
Results of research of working capability of refined pipelayer equipment
}

\author{
Korchagin P.A. \\ Dept. of Scientific Research \\ Siberian Automobile and Highway University (SibADI) \\ Omsk, Russia \\ Korchgin_PA@mail.ru
}

\author{
Letopolskiy A.B. \\ Dept. of Scientific Research \\ Siberian Automobile and Highway University (SibADI) \\ Omsk, Russia \\ Antoooon-85@mail.ru
}

\author{
Teterina I.A. \\ Dept. of Scientific Research \\ Siberian Automobile and Highway University (SibADI) \\ Omsk, Russia \\ iateterina@mail.ru
}

\begin{abstract}
The paper proposes a new variant of additional support improvement of the pipelayer boom. The supposed technical solution allows one to increase smoothing and stability of pipelayer operation. The result is achieved by adding extra elements into the construction of the pipelayer boom support. The proposed construction presupposes that cargo boom will be joined with the pipelayer support, which consists of a hydraulic cylinder and a metal base, used for cylinder fixation to the support with bolted-type connection. The relations, gained from the computed pattern of the pipelayer working equipment, are given to prove the efficiency of the proposed design solution. The calculated relations allow defining the size of the loads applied on the pipelayer. The results of the theoretical research, carried out in Solid works, are demonstrated in graphics, and it shows the stresses, shifts and deformations in boom design of the pipelayer used for long-distance pipeline placement. The additional advantage is that the size of the strength margin in the support structure of the pipelayer boom was obtained due to the conducted research.
\end{abstract}

Keywords-pipelayer, pipeline construction, road building machines, long-distance pipeline, pipelayer boom, working equipment.

\section{INTRODUCTION}

Pipelayer is a narrow focus road building machine used in pipeline construction and repair [1,2]. Pipelayer is a lift crane equipped with a side boom. The pipelayer lifting capacity of the same load in different boom inclinations is not stable. The pipelayer in its core function is primarily subjected to the external vertical load applied to its hoist hook. It refers to the weight of break-bulk rigid cargo or to the weight of the lifted elastic section of the pipeline [3]. The second variant of external stresses has a complex character, because it depends not only on lifted pipeline but on the deflection shape [3].

Pipelayer movement on uneven microrelief and uncoordinated actions of operators in machine teamwork with general cargo lead to the constant change of pipeline deflection form in vertical plane and to the mass redistribution of upper section between the machines [4]. In other words, if you work with a single rigid cargo, the stress will be constant and will depend only on the mass of the load. In pipeline construction, the stress has a changeable character, because it depends on constantly changing technological factors. First of all, it depends on the weight of the section and on other pipeline characteristics (Fig.1) [3].

In order to provide working safety during pipeline construction, it is supposed to improve the structure of the working equipment.

\section{TASK ASSIGNMENT}

In working conditions, the loads applied to the construction equipment often does not suit the machine load capacity. In this case, there is a danger of pipelayer tipping [2]. One of the problem solution is a support, mounted on the main pipelayer boom (Fig. 2) [4].

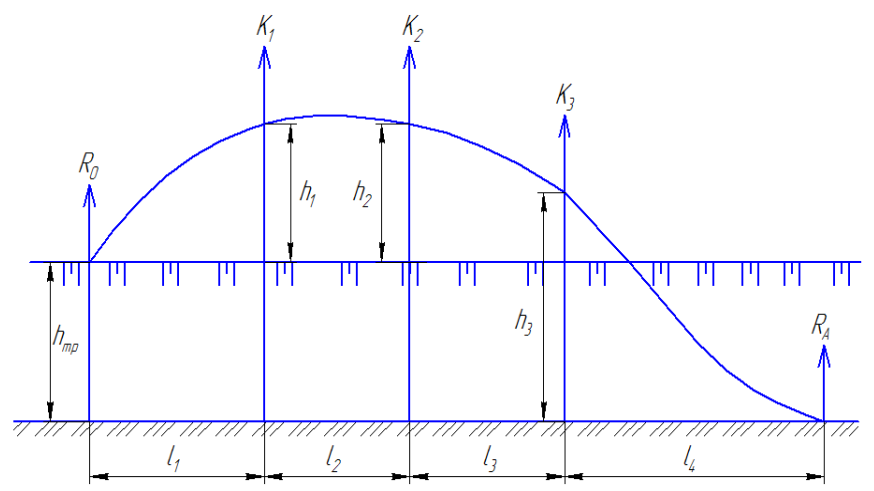

Fig.1. Technological scheme of pipeline construction. 


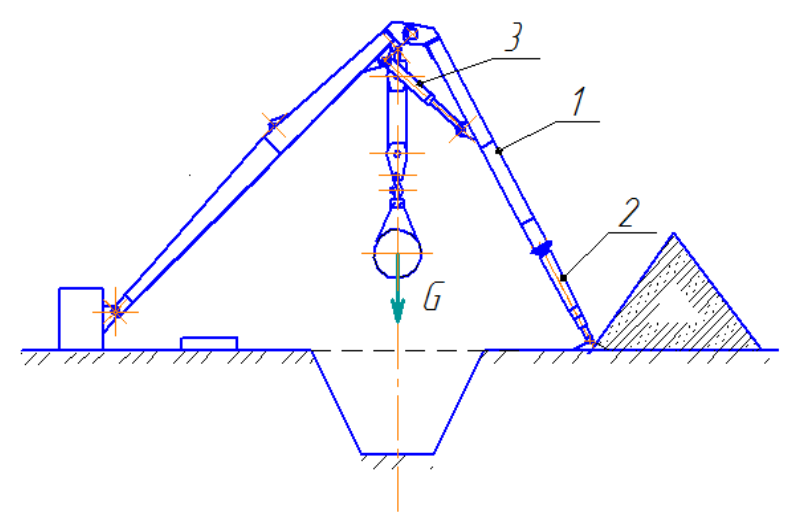

Fig.2. Pipelayer boom equipped with support: 1-support; 2-hydaulic cylinder; 3- hydraulic cylinder of support extension.

Pipelayer is a crane with a side boom. Its capability to carry the same load in some variants of the boom angles is not stable. In almost vertical position, the pipelayer is able to lift more load. The more the boom length, the more risk of rolling over towards the load [3].

To define the load size influence on the pipelayer hook, the calculation of the ditch characteristics and of the minimum number of pipelayers needed for the operation has been made [5].

Ditch depth $\left(\mathrm{h}_{\mathrm{tr}}\right)$ :

$\mathrm{h}_{\mathrm{tr}}=0.8+\mathrm{D}_{\mathrm{tr}}$

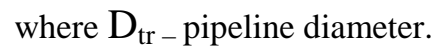

Ditch width:

$$
B_{\text {tr }}=1.5 \cdot D_{\text {tr }}
$$

The optimal distance between the suspension points is calculated according to the formulas:

$$
\begin{aligned}
& l_{1}=2.46 \cdot \sqrt[4]{\frac{E I h_{1}}{q}}, \\
& l_{2}=1.22 \cdot \sqrt[4]{\frac{E I h_{1}}{q}}, \\
& l_{3}=1.22 \cdot \sqrt[4]{\frac{E I h_{3}}{q}} \\
& l_{4}=2.46 \cdot \sqrt[4]{\frac{E I h_{3}}{q}}
\end{aligned}
$$

where $E I$ - rigidity of the pipeline being built; $q$ - weight of 1 meter of the pipeline; $h_{1}, h_{2}-$ lift height of the pipeline $\left(h_{l}=h_{2}, h_{3}=\mathrm{h}_{\mathrm{tr}}+0.5\right)$.
The stress (Fig.1) influenced on the pipelayer was computed by formulas [5]:

$$
\begin{aligned}
& K_{1}=q \cdot\left(\frac{2}{3} \cdot l_{1}+\frac{1}{2} \cdot l_{2}\right), \\
& K_{2}=q \cdot\left(\frac{1}{2} \cdot l_{2}+\frac{1}{2} \cdot l_{3}\right), \\
& K_{3}=q \cdot\left(\frac{1}{2} \cdot l_{3}+\frac{2}{3} \cdot l_{4}\right) .
\end{aligned}
$$

The analysis of the results got for load calculations at the suspension points for the pipelayer TG - 124 (Table 1) allows us conclude that in operational process one suspension point is subjected to the load $93742 \mathrm{~N}$, it does not correspond to the loading capacity of the pipelayer, which is equal to $67000 \mathrm{~N}$ with the hook radius $3.96 \mathrm{~m}[1,2]$.

\section{TABLE 1. LOAD CAPACITY OF THE PIPELAYER TG-124}

\begin{tabular}{|l|l|l|l|l|l|}
\hline $\begin{array}{l}\text { Hook radius (from the left edge } \\
\text { of rolling over) }\end{array}$ & 1.5 & 2.5 & 3.5 & 4.5 & 5.6 \\
\hline $\begin{array}{l}\text { Load capacity with } \\
\text { counterweight and stability } \\
\text { coefficient 1.4 tons }\end{array}$ & 12.5 & 10.9 & 7.6 & 5.75 & 4.6 \\
\hline
\end{tabular}

The boom structure of the upgraded pipelayer consists of two parts: the main boom and the additional support fixed with none rigid joint [5]. The distinctive feature of the construction is that the boom support is made in the form of suspended hydraulic cylinder pivotally connected with the block [5,6]. As a hydraulic cylinder, it is supposed to use a standard hydraulic cylinder 55111-8603010 [1,4].

\section{THEORY (RESEARCH DATA AND METHODS)}

Fig. 3 shows the pipelayer boom equipped with the support. It demonstrates the acting reaction power of the mounting surface and stresses arising during pipeline construction [7].

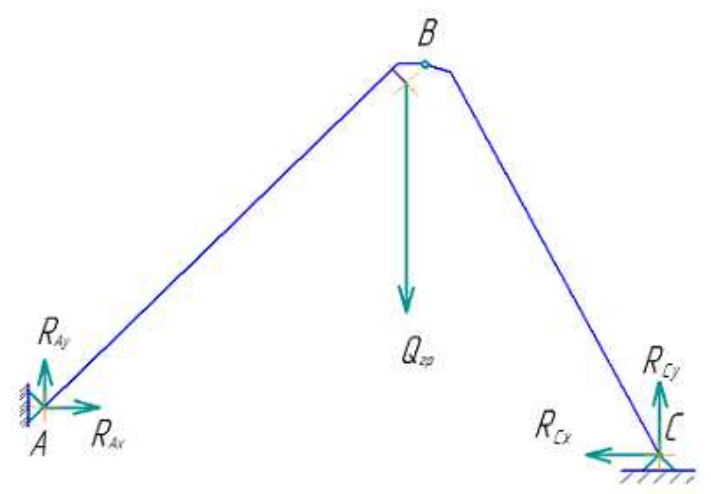

Fig.3. Stresses and reactions effected the boom. 
To calculate $R_{A x}, R_{A y}, R_{C x}$ and $R_{C y}$, it is necessary to divide the structure in two parts (Fig. 4,5) in accordance with non-rigid connection in the hinge $\mathrm{B}$. The value of each equilibrium is found by equilibrium equation of forces. As the result, new reactions $R_{B x}$ and $R_{B y}$ were discovered in the hinge B (Fig. 5).

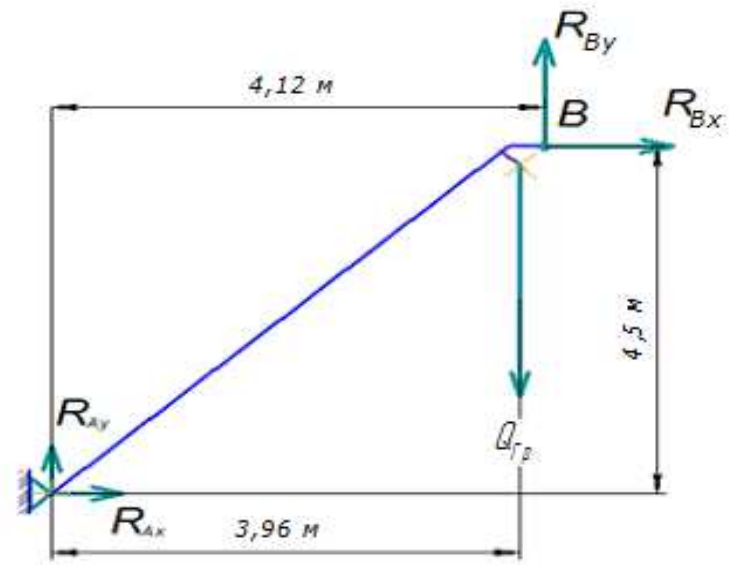

Fig.4. Left section of the structure.

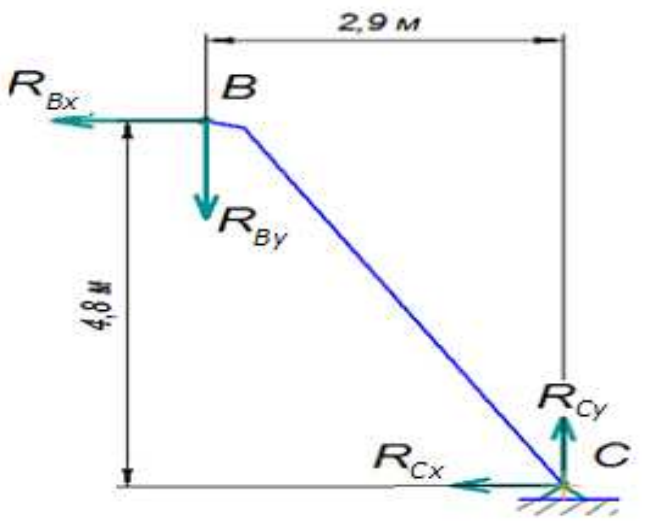

Fig.5. Right section of the structure.

To calculate unknown stress influence, three equations were introduced: sum of forces in relation to axis $\mathrm{X}$; sum of forces in relation to axis $\mathrm{Y}$; the sum of moments in relation to point A [8,9]:

$\sum F_{i X}=R_{A x}+R_{B x}=0$,

$\sum F_{i Y}=R_{A y}+R_{B y}-Q_{z p}=0$,

$\sum M_{A}=R_{B y} \cdot 4.12-R_{B x} \cdot 4.5-Q_{2 p} \cdot 3.96=0$.

A stress balance equation was composed: sum of forces in relation to axis X; sum of forces in relation to axis $\mathrm{Y}$; sum of moments in relation to point $\mathrm{B}$, the sum of moments in relation to point $\mathrm{C}[10,11]$ :

$\sum F_{i X}=-R_{B x}-R_{C x}=0$,

$$
\begin{aligned}
& \sum F_{i Y}=R_{C y}-R_{B y}=0, \\
& \sum M_{C}=R_{B x} \cdot 4.8+R_{B y} \cdot 2.9=0, \\
& \sum M_{B}=-R_{C x} \cdot 4.8+R_{C y} \cdot 2.9=0 .
\end{aligned}
$$

From the formula (13) $R_{B x}=-R_{C x}$.

From the formula (14) $R_{B y}=R_{C y}$.

From the formula (16) $R_{C y}=\frac{R_{C x} \cdot 4.8}{2.9}$.

Having added equations (17) and (19) into formula (12), reaction $R_{C X}$ was defined:

$$
\frac{R_{C x} \cdot 4.8}{2.9} \cdot 4.12+R_{C x} \cdot 4.5-Q_{2 p} \cdot 3.96=0 .
$$

The resultant reaction force $R_{B x}$ and $R_{B y}$ arising in joint is equal to algebraic sum of these forces:

$$
R_{B}=\sqrt{R_{B x}^{2}+R_{B y}^{2}} .
$$

Flexural moment in the hinge pin [11]:

$$
M_{\Pi}=\frac{N \cdot a}{2},
$$

where $N$ - cross bending force, $\mathrm{kN}$.

$a$ - distance from eye to the point with applied force, $\mathrm{cm}$.

Minimum resistance moment of the axis cross section [12]:

$$
W_{\Pi}=\frac{M_{\Pi}}{m \cdot 0.1 \cdot R}
$$

where $m$ - coefficient of the working conditions;

$R$ - calculated resistance of the rolling steel, $\mathrm{mPa}$.

An axis diameter was defined by the formula [13]:

$$
d=\sqrt[3]{10 \cdot W_{\Pi}}
$$

For the further calculation, it is needed to check on the axis cut. It may be done by using formula $[5,14]$ :

$$
\frac{N}{n_{c p} \cdot \pi \cdot \frac{d^{2}}{4}}<m \cdot R_{c p},
$$


Where $n_{c p}$ - number of axis cuts, $n_{c p}=2$;

$R_{c p}$ - shear strength, MPa.

\section{EXPERIMENTAL RESULTS (RESEARCH RESULTS)}

This theoretical research was carried out in Solid Works to define strength characteristics of the proposed pipelayer boom structure with additional support $[5,15]$.

Strength calculations were conducted in the following order [8]:

1. To set the material and to define fixation type for the boom and its hydrocylinder (fixation type for the boom and its hydrocylinder - fixed hinge, impact of soil on the support - fixed geometry).

2. To set the load (the load is directed down along the rope. Cylindrical shape was taken as a rape model, mounted in the eyelets of the hook clip. The force is acted on the structure surface with opposite direction).

3. To construct the net on a solid body dividing the structure into smaller segments.

4. To make calculations [9].

Fig. 6 represents the visualization of the theoretical research intended to calculate stresses arising in the structure. Minimum force values are equal to $0.257 \mathrm{~Pa}$; maximum force values are equal to $2.63 \cdot 10^{8} \mathrm{~Pa}$.

The conducted research allowed one to define the sections of the maximum stress accumulation in the support structure at prescribed load equivalent to 12 tons $[15,16]$. The study showed that maximum stress does not exceed the acceptable strength level of the material $[4,17]$.

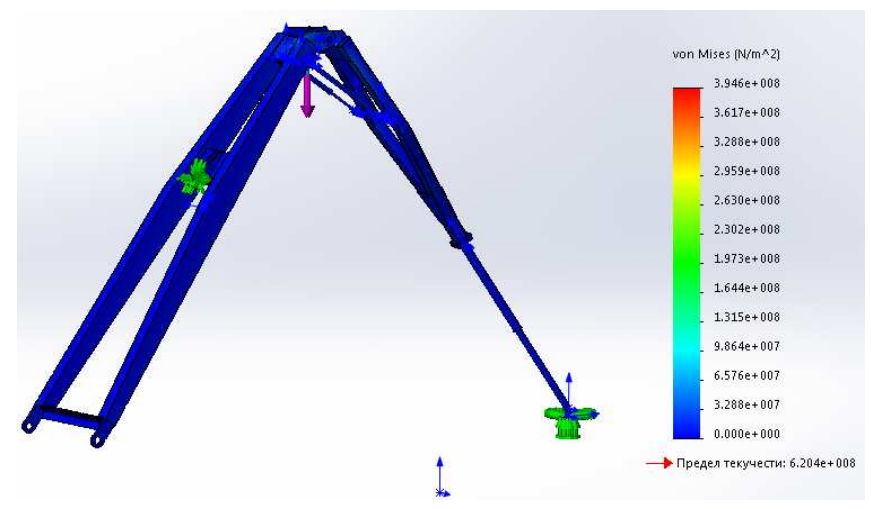

Fig.6. Research of the stresses in the support structure of the pipelayer boom.

Fig. 7 gives the visualization of the theoretical research intended to calculate shear in the structure of the pipelayer boom support. The studies revealed that minimum shear value is $0 \mathrm{~mm}$, maximum is $4.062 \mathrm{~mm}$.

The theoretical research of the shears allowed to identify areas with possible detail displacement in the support structure. It was found, that the maximum shears are concentrated at the attachment point of the support to the hydrocylinder. Avoiding of possible displacement may be achieved either by increasing the number of bolted joints or by increasing the number of fastening elements.

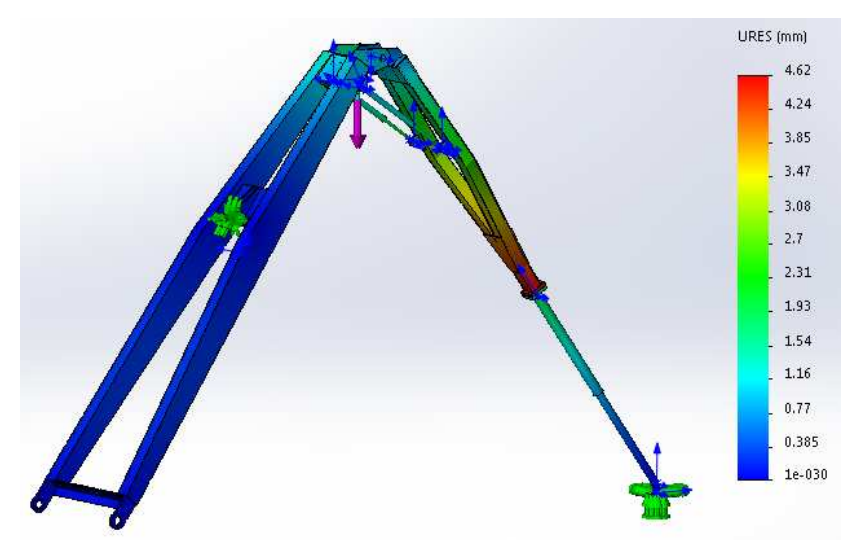
boom.

Fig.7. Research of the shear in the support structure of the pipelayer

Fig. 8 introduces the visualization of the theoretical research intended to define deformation dimension arising in the structure of the pipelayer boom support $[5,18]$. Under the load equal to 12 tons the minimum deformation value is $1.93 \cdot 10^{-7} \mathrm{~mm}$, maximum is $5.8 \cdot 10^{-4} \mathrm{~mm}$.

The deformation study allowed to determine the possible deformation areas in the proposed structure of the pipelayer boom support. The conducted research let us conclude that critical limits in maximum possible load capacity modelling do not arise in equipment units [5].

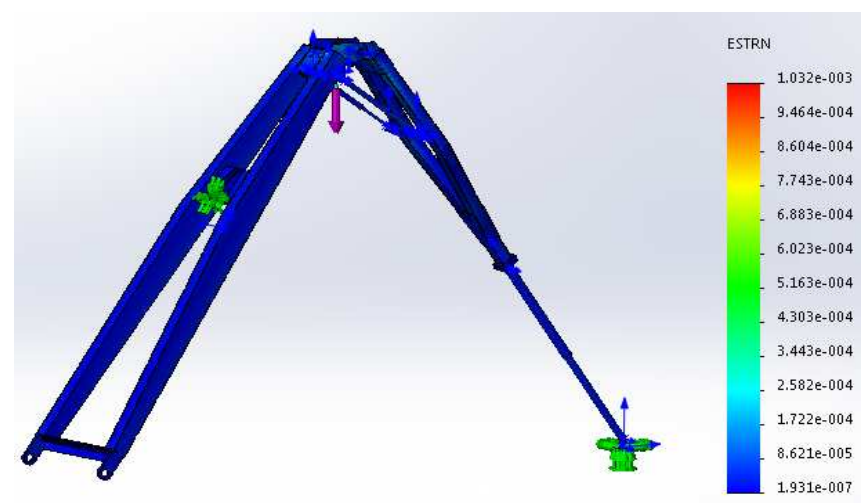

Fig.8. Research of the deformations in the support structure of the pipelayer boom.

Fig. 9 represents the visualization of the theoretical research intended to compute the safety factor in the structure of the pipelayer boom support $[5,16]$. The minimum safety factor is 1.6 , maximum is 4.76 .

The study of the safety factor allowed us to establish strength characteristics at every point of the proposed structure as well as to define whether the structure is capable to withstand the given loads or not in accordance with factor of safety (FOS). In order to withstand the given loads, the safety 
coefficient has to be more than 1; that is why the structural elements has to be made with safety coefficient more than 1.5.

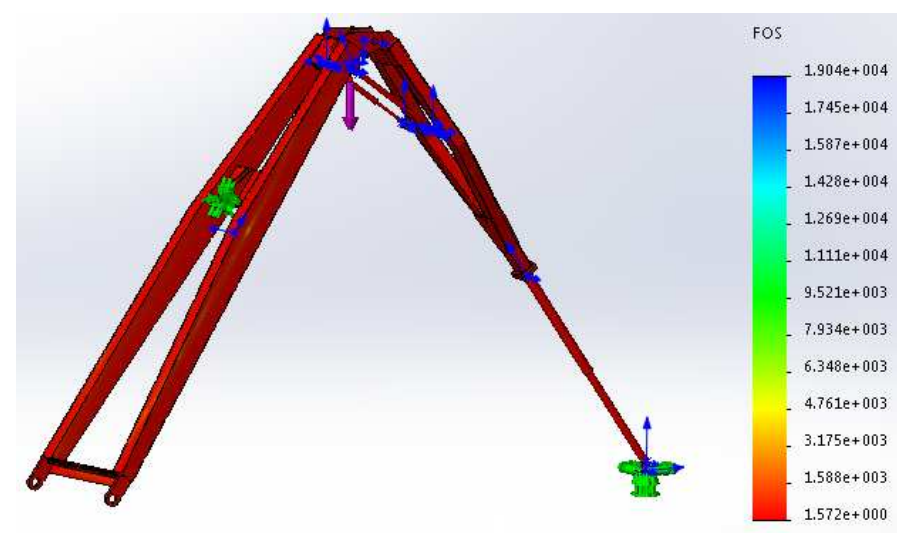

Fig.9. Research of the safety factor in the support structure of the pipelayer boom.

The application of the pipelayer with the additional support can reduce the amount of the equipment used in the construction of the pipeline. The supposed support structure will allow one to increase the smoothness of operation, to improve the pipelayer stability and to make the pipeline construction process much safer.

\section{V.CONCLUSION}

In the conducted research, the calculated dependences were obtained for the stress computation (stresses arising in joint connections). The resultant formulas allow computing the stress change, which depends on the mass of the pipe laid into the ditch. It was discovered that arising stresses and displacements in the structure of the working equipment do not exceed the critical level. The strength analyses allow us to conclude that the structure of the pipelayer working equipment has the sufficient strength level.

The application of the pipelayer with the additional support can reduce the amount of the equipment used in the construction of the pipeline. The supposed support structure will allow one to increase the smoothness of operation, to improve the pipelayer stability and to make the pipeline construction process much safer.

\section{References}

[1] "Welded steel pipes for the gas, oil and product pipelines. Introduced in 2002-01-01”, GOST standard R 52079 -2003, Moscow: Gosstandard of Russia, 2005.

[2] V. Usanov, A. Berezin, A. Vorontsov, V. Zhutkin, Yu. Babakov, "Cost reduction in pipelayer crane operation", Technical control, vol.11 (108), pp.686-687, 2015.

[3] D. Parshin, M. Shoshiashvili "Automatic stability control of the pipelayer", J. Building and road making machines, vol.10, pp.16-18, 1990.

[4] A. Voronin, V. Lipskiy, P. Serenkov, "Accident rate analyses of the long distance pipeline in Belarus, Russia, Europe and in the USA", J. Vestnik of Polotskiy state university, ed. F., Construction. Applied science, vol. 16, pp.69-74, 2012.

[5] A. Voronin, V. Lipskiy, "Process description of long distance pipiline transportation by using functional modelling system", Security and reliability of the pipeline transportation, Polotsk, 2014, pp. 49-51.

[6] I. Vaschuk, V. Utkin, B. Kharkun, "Pipelayers", Machine building, Moscow, 1989, p. 124.

[7] Yu. Dudoladov, "Pipelayer cranes", Moscow, 1981, p.100.

[8] A. Letopolskiy, D. Semkin,A. Kovalev, "Construction technics and technologies", vol.2, 2016, p.11.

[9] I. Teterina, "Efficiency improvement of the vibroprotection system for sweeping machine operator", Omsk, 2017, p. 18.

[10] S. Dobronravov, M. Dobronravov, "Construction machines and equipment", $2^{\text {nd }}$ ed., Moscow, 2006.

[11] D. Chernyavskiy, D. Chernyavskaya, "Optimal parameter calculation of the shock rotational impulse in concrete drilling with rotary hammer", J. Omskiy Vestnik, vol.137, pp. 44-46, 2015.

[12] L.Denisova, V. Mescherikov, "Automated parameter synthesis of the operation system made by using genetic algorithm", J. Automation and remote control,vol. 76 (1), pp.149-156, 2015.

[13] I. Boyarkina, V. Tarasov, "Regularities of the working equipment element mass reduction in the hydraulic power cylinder piston for the bucket boom machine size standard", J. of Physics, vol. 858 (1), p.120, 2017.

[14] V. Scherbakov, V. Koritv, E. Shershneva, "Obstacle influence on stress displacement in gantry crane operation", J. Russian engineering research, vol. 36 (3), pp.194-197, 2016.

[15] A. Rannev, M. Polosin, "Structure and operation of road constrution machines", $3^{\text {rd }}$ ed., Moscow, 2005, pp. 230-236.

[16] V. Zarubin, "Pipelayer", Moscow, 1984, pp. 100-104.

[17] V. Minaev, "Machinery for a long distance pipeline construction", $2^{\text {nd }}$ ed., Moscow, 1985, pp. 57-59.

[18] B. Kharkun, O. Vereynov,, V. Utkin,, V. Shevelenko, V. Sliskov, B. Zakharchuk, "Pipilayer crane", Patent 703490 owned by Scientific research institute of building and road constuction machinery, vol. 46, 1978. 